Elect. Comm. in Probab. 16 (2011), 732-740

\title{
REGULAR G-MEASURES ARE NOT ALWAYS GIBBSIAN
}

\author{
ROBERTO FERNÁNDEZ
}

Department of Mathematics, University of Utrecht, P.O.box 80010, NL-3508 TA Utrecht, The Netherlands

email: R.Fernandez1@uu.nl

SANDRO GALLO

Instituto de Matemática, Estatística e Computação Científica, Universidade Estadual de Campinas, Rua Sergio Buarque de Holanda, 651 13083-859 Campinas, Brasil

email: gsandro@ime.unicamp.br

GRÉGORY MAILLARD

CMI-LATP, Aix Marseille Université, 39 rue F. Joliot-Curie, F-13453 Marseille Cedex 13, France

email: maillard@cmi.univ-mrs.fr

Submitted June 21, 2011, accepted in final form October 13, 2011

AMS 2000 Subject classification: Primary 60G10, 82B20; Secondary 37A05.

Keywords: Discrete-time stochastic processes, $g$-measures, chains with complete connections, nonGibbsianness, chains with variable-length memory.

Abstract

Regular $g$-measures are discrete-time processes determined by conditional expectations with respect to the past. One-dimensional Gibbs measures, on the other hand, are fields determined by simultaneous conditioning on past and future. For the Markovian and exponentially continuous cases both theories are known to be equivalent. Its equivalence for more general cases was an open problem. We present a simple example settling this issue in a negative way: there exist $g$-measures that are continuous and non-null but are not Gibbsian. Our example belongs, in fact, to a well-studied family of processes with rather nice attributes: It is a chain with variable-length memory, characterized by the absence of phase coexistence and the existence of a visible renewal scheme.

\section{Introduction}

Measures on $E^{\mathbb{Z}}$ are the object of two very developed theories. On the one hand, the theory of chains of complete connections, started in [16] and developed under a variety of names in slightly non-equivalent frameworks: chains of infinite order [10], g-measures [12], uniform martingales [11], etc. On the other hand, the theory of one-dimensional Gibbs measures started in [2, 15] and whose classical reference is the treatise [9]. The former theory interprets $\mathbb{Z}$ as discrete time 
and measures as discrete-time processes. The building blocks of the theory are, therefore, transition probabilities, that is, conditional probabilities with respect to the past. Chains, $g$-measures, etc are defined by their invariance under - or consistency with - these transition probabilities. In contrast, Gibbsianness refers to fields in a spatial setting determined by distributions on finiteregions conditioned on exterior configurations. In one dimension, if $\mathbb{Z}$ is interpreted as time, this corresponds to conditioning both with respect to the past and to the future. Of course, this is only part of the story. Both processes and Gibbs measures are required to satisfy suitable regularity conditions, as reviewed below.

Given this state of affairs it is natural to wonder about the relation of both theories. Are they equivalent? Does one-side conditioning carry the same information as two-side conditioning? Is every regular process a Gibbs measure and vice-versa? To be sure, there is another source of difference related to non-nullness. The standard theory of Gibbs measures deals with systems with no forbidden configurations. Many important instances of processes, on the other hand, include grammars or local exclusion rules (subshifts of finite type). The previous questions should be stated, then, in the common non-null framework. In this set-up, the equivalence of both theories has long been known to be true for Markov processes and fields (see, for instance, [9, Chapter 11]) and when continuity rates are exponentially decreasing [6]. In this note we exhibit a simple example - where all calculations can be explicitly performed - showing that this equivalence is not true in general.

In more detail, below we construct a $g$-measure $\mu$ on $\{0,1\}^{\mathbb{Z}}$ with the following properties:

- $\mu$ is non-null: it gives nonzero measure to every cylinder.

- There exists a continuous $g$ function for which $\mu$ is the only consistent measure.

- $\mu$ is left-right symmetric [as proven in the third line of (3.12)].

- $\mu$ is a variable-length memory chain which admits a renewal construction with visible renewals (see [8] for example).

- $\mu$ can be perfectly simulated (for example by the method of [8]).

Yet, despite all these fine properties, the measure $\mu$ is non-Gibbsian.

This example shows that regular $g$-measures are a different type of creature than Gibbsian measures and respective theories (large-deviations, uniqueness theorems, variational approach) can not, in general, be imported from one to the other. In particular, readers are warned that a $g$ function of the form $g=\mathrm{e}^{\phi}$ with $\phi$ "nice" does not automatically deserve the qualifier "Gibbsian". These observations complement previous studies on differences between one-sided and two-sided measurability done in the more general framework of ergodic theory (see, e.g. [4] and references therein).

Another potential source of confusion arises from the traditional use, by people working in dynamical systems, of the word "Gibbsian" to refer to SRB measures (Sinai-Ruelle-Bowen measures, see e.g. [17] or [1]). As briefly reviewed below, the set of non-null SRB measures is strictly contained in the set of statistical mechanical Gibbs measures (but the former, unlike the latter, can also incorporate exclusions and subshifts). Hence our $g$-measure $\mu$ is also non-Gibbsian in SRB sense.

Let us conclude with a brief explanation of the non-Gibbsianness argument below. The measure $\mu$ is non-null and consistent with a continuous $g$-function. This means that, upon conditioning, 
it becomes asymptotically insensitive to the far past. In order to be Gibbs, the same asymptotic insensitivity must hold but simultaneously with respect to past and future. More precisely, in our example, the continuity of the $g$-function is guaranteed by the fact that the probability of having a 1 conditioned on a large string of zeros converges, as the first 1 recedes to $-\infty$. For the measure to be Gibbsian this same continuity must hold for two-side conditioning. A delicate case arises, however, when conditioning on having the all-zero configuration both towards the past and the future. Of course, this is an impossible configuration for $\mu$, so that the actual value of this conditional probability is irrelevant. Rather, the Gibbsianness question refers to whether conditional probabilities converge (to whatever) as both the first 1 to the left and the first 1 to the right move away. This is an essential property in the sense that its absence can not be fixed by measure-zero redefinitions. Theorem 3.1 shows that for some $g$-functions this two-side continuity is impossible. As an aside, we prove that the "all-0" configuration is the only point of discontinuity of the two-side conditional probabilities. The measure $\mu$ is, therefore, almost Gibbsian and thus weakly Gibbsian (see, for instance, [5, Section 4.4] for the corresponding definitions and historical references to these notions).

\section{Preliminaries}

We consider a measurable space $(E, \mathscr{E})$ where $E=\{0,1\}$ is a two-symbol alphabet and $\mathscr{E}$ is the associated discrete $\sigma$-algebra. We denote by $(\Omega, \mathscr{F})$ the associated product measurable space, that is $\Omega=E^{\mathbb{Z}}, \mathscr{F}=\mathscr{E}^{\mathbb{Z}}$. For each $\Lambda \subset \mathbb{Z}$ we denote $\Omega_{\Lambda}=E^{\Lambda}$ and $\sigma_{\Lambda}$ for the restriction of a configuration $\sigma \in \Omega$ to $\Omega_{\Lambda}$, namely the family $\left(\sigma_{i}\right)_{i \in \Lambda} \in E^{\Lambda}$. Also, $\mathscr{F}_{\Lambda}$ will denote the sub$\sigma$-algebra of $\mathscr{F}$ generated by cylinders based on $\Lambda\left(\mathscr{F}_{\Lambda}\right.$-measurable functions are insensitive to configuration values outside $\Lambda$ ). When $\Lambda$ is an interval, $\Lambda=[k, n]$ with $k, n \in \mathbb{Z}$ such that $k \leq n$, we use the notation: $\omega_{k}^{n}=\omega_{[k, n]}=\omega_{k}, \ldots, \omega_{n}, \Omega_{k}^{n}=\Omega_{[k, n]}$ and $\mathscr{F}_{k}^{n}=\mathscr{F}_{[k, n]}$. For semi-intervals we denote also $\mathscr{F}_{\leq n}=\mathscr{F}_{(-\infty, n]}$, etc. The concatenation notation $\omega_{\Lambda} \sigma_{\Delta}$, where $\Lambda \cap \Delta=\emptyset$, indicates the configuration on $\Lambda \cup \Delta$ coinciding with $\omega_{i}$ for $i \in \Lambda$ and with $\sigma_{i}$ for $i \in \Delta$.

We start by briefly reviewing the well-known notions of chains in a shift-invariant setting. In this particular case, chains are also called $g$-measures (see [12]).

Definition 2.1. $A$ regular $g$-function $P$ on $\Omega$ is a probability kernel $P: E \times \Omega_{-\infty}^{-1} \rightarrow[0,1]$, i.e.,

$$
\sum_{\omega_{0} \in E} P\left(\omega_{0} \mid \omega_{-\infty}^{-1}\right)=1 \quad \forall \omega_{-\infty}^{-1} \in \Omega_{-\infty}^{-1}
$$

such that:

- the function $P\left(\omega_{0} \mid \cdot\right)$ is continuous for each $\omega_{0} \in E$, i.e., for all $\epsilon>0$ there exists $n \geq 0$ so that

$$
\left|P\left(\omega_{0} \mid \omega_{-\infty}^{-1}\right)-P\left(\omega_{0} \mid \sigma_{-\infty}^{-1}\right)\right|<\epsilon
$$

for all $\omega_{-\infty}^{0}, \sigma_{-\infty}^{0} \in \Omega_{-\infty}^{0}$ with $\omega_{-n}^{0}=\sigma_{-n}^{0}$;

- the function $P\left(\omega_{0} \mid \cdot\right)$ is strongly non-null for each $\omega_{0} \in E$, i.e., $P\left(\omega_{0} \mid \cdot\right) \geq c>0$.

[Property (2.2) is indeed continuity with respect to the product discrete topology of $\Omega$. By a compactness argument, this implies uniform continuity.] 
Definition 2.2. A probability measure $\mu$ on $(\Omega, \mathscr{F})$ with underlying process $\left(X_{i}\right)_{i \in \mathbb{Z}}$ on $(\Omega, \mathscr{F}, \mu)$ is said to be a regular $g$-measure if $\mu$ is shift-invariant and there exists a regular $g$-function $P$ such that $\mu$ is consistent with $P$, namely,

$$
\mu\left(X_{0}=\omega_{0} \mid X_{-\infty}^{-1}=\omega_{-\infty}^{-1}\right)=P\left(\omega_{0} \mid \omega_{-\infty}^{-1}\right)
$$

for all $\omega_{0} \in E$ and $\mu$-a.e. $\omega_{-\infty}^{-1} \in \Omega_{-\infty}^{-1}$.

Remark 2.3. In the consistency definition (2.3), $\mu$ needs only to be defined on $\left(\Omega_{-\infty}^{0}, \mathscr{F}_{\leq 0}\right)$. By shift-invariance, $\mu$ can be extended in a unique way to $(\Omega, \mathscr{F})$. Thus, without loss of generality, we identify $\mu$ on $\left(\Omega_{-\infty}^{0}, \mathscr{F}_{\leq 0}\right)$ with its natural extension on $(\Omega, \mathscr{F})$.

The previous definition is not very useful to prove the regularity of a measure. For this, the following well known result is often useful. Let us call a measure $\mu$ on $(\Omega, \mathscr{F})$ non-null if it gives non-zero measure to every cylinder. We then have (see, for instance, [11]):

Theorem 2.4. A probability measure $\mu$ on $(\Omega, \mathscr{F})$ with underlying process $\left(X_{i}\right)_{i \in \mathbb{Z}}$ on $(\Omega, \mathscr{F}, \mu)$ is a regular $\mathrm{g}$-measure iff it is non-null, shift-invariant and the sequences

$$
\left[\mu\left(X_{0}=\omega_{0} \mid X_{-n}^{-1}=\omega_{-n}^{-1}\right)\right]_{n \geq 1} \text { converge uniformly as } n \rightarrow \infty .
$$

Let us now review the notions of specification and Gibbs measures. It involves definitions and properties analogous to the preceding ones, but replacing one-side by two-side conditioning. (We specialize to the one-dimensional setting but similar notions and results are valid for any dimension.)

Definition 2.5. A specification $\gamma$ on $(\Omega, \mathscr{F})$ is a family of probability kernels $\left\{\gamma_{\Lambda}: \Lambda \subset \mathbb{Z},|\Lambda|<\infty\right\}$, $\gamma_{\Lambda}: \mathscr{F} \times \Omega \rightarrow[0,1]$ such that for all finite $\Lambda \subset \mathbb{Z}$ :

- $\gamma_{\Lambda}(A \mid \cdot) \in \mathscr{F}_{\Lambda^{\mathrm{c}}}$, for each $A \in \mathscr{F}$;

- $\gamma_{\Lambda}(B \mid \omega)=\mathbb{1}_{B}(\omega)$, for each $B \in \mathscr{F}_{\Lambda^{\mathrm{c}}}$ and $\omega \in \Omega$;

- $\gamma_{\Delta} \gamma_{\Lambda}=\gamma_{\Delta}$, for each finite $\Delta \subset \mathbb{Z}: \Delta \supset \Lambda$, i.e.,

$$
\iint h(\xi) \gamma_{\Lambda}(d \xi \mid \sigma) \gamma_{\Delta}(d \sigma \mid \omega)=\int h(\sigma) \gamma_{\Delta}(d \sigma \mid \omega)
$$

for all measurable functions $h$ and configurations $\omega \in \Omega$.

In words, a specification is almost a regular system of conditional probabilities for finite regions conditioned on the external sigma algebras. The only difference lies in the fact that conditional probabilities satisfy (2.5) for almost all $\omega$ with respect to some measure known beforehand. In the definition of specification there is no previously known measure, thus condition (2.5) is asked for all $\omega$ (see e.g. [3] for more details). We can now define regularity, as expected, as a property of all the kernels $\gamma_{\Lambda}$. In our setting, however, we are interested only in non-null specifications and they, in fact, are entirely determined by their single-site part $\left\{\gamma_{\{i\}}: i \in \mathbb{Z}\right\}$ (see [7] and references therein). Relevant definitions need only be done, thus, at the level of these single-site kernels. That is what we do now, to emphasize the parallelism with the treatment of $g$-measures. For brevity we denote $\gamma_{\{i\}}\left(\omega_{i} \mid \cdot\right)$ the kernel $\gamma_{\{i\}}$ applied to the cylinder of base $\omega_{i}$. 
Definition 2.6. A specification $\gamma$ on $(\Omega, \mathscr{F})$ is regular if, for each $i \in \mathbb{Z}$,

- the function $\gamma_{\{i\}}\left(\omega_{i} \mid \cdot\right)$ is continuous for each $\omega_{i} \in E$, i.e., for all $\epsilon>0$, there exists $n, m \geq 0$ so that

$$
\left|\gamma_{\{i\}}\left(\omega_{i} \mid \omega_{\{i\}^{c}}\right)-\gamma_{\{i\}}\left(\omega_{i} \mid \sigma_{\{i\}^{c}}\right)\right|<\epsilon
$$

for all $\omega, \sigma \in \Omega$ with $\omega_{-n}^{m}=\sigma_{-n}^{m}$;

- the function $\gamma_{\{i\}}\left(\omega_{i} \mid \cdot\right)$ is strongly non-null for each $\omega_{i} \in$ E, i.e., $\gamma_{\{i\}}\left(\omega_{i} \mid \cdot\right) \geq c>0$.

Definition 2.7. A probability measure $\mu$ on $(\Omega, \mathscr{F})$ with underlying field $\left(X_{i}\right)_{i \in \mathbb{Z}}$ on $(\Omega, \mathscr{F}, \mu)$ is said to be a Gibbs measure if $\mu$ is consistent with some regular specification $\gamma$, namely,

$$
\mu\left(X_{i}=\omega_{i} \mid X_{\{i\}^{\mathrm{c}}}=\omega_{\{i\}^{\mathrm{c}}}\right)=\gamma_{\{i\}}\left(\omega_{i} \mid \omega_{\{i\}^{\mathrm{c}}}\right)
$$

for all $\omega_{i} \in E$ and $\mu$-a.e. $\omega_{\{i\}^{c}} \in \Omega_{\{i\}^{c}}$.

The following is the analogues of Theorem 2.4.

Theorem 2.8. A shift-invariant probability measure $\mu$ on $(\Omega, \mathscr{F})$ with underlying field $\left(X_{i}\right)_{i \in \mathbb{Z}}$ on $(\Omega, \mathscr{F}, \mu)$ is a Gibbs measure iff it is non-null and the sequences

$$
\left[\mu\left(X_{0}=\omega_{0} \mid X_{-n}^{-1}=\omega_{-n}^{-1}, X_{1}^{m}=\omega_{1}^{m}\right)\right]_{n, m \geq 1} \quad \text { converge uniformly as } n, m \rightarrow \infty .
$$

A measure violating (2.8) is, thus, a non-Gibbsian measure (see, e.g. [5] and references therein). More specifically, the absence of convergence of a sequence (2.8) corresponds to an essential discontinuity at $\omega \in \Omega$, that is, a discontinuity in the conditional expectations that can not be removed by a redefinition on a zero-measure set (a more detailed discussion of these issues can be found in [5, Section 5.3]).

The link between Definition 2.7 and the usual notion in classical statistical mechanics is provided by a theorem due to Kozlov [14] that states that a specification is Gibbsian if and only if it has the Boltzmann form

$$
\gamma_{\Lambda}\left(\omega_{i} \mid \omega_{\{i\}^{c}}\right)=\exp \left\{-\sum_{A \ni i} \phi_{A}\left(\omega_{A}\right)\right\} / \text { Norm. }
$$

where the functions $\phi_{A}$ (interaction) satisfy the summability condition

$$
\sup _{i \in \mathbb{Z}} \sum_{A \ni i}\left\|\phi_{A}\right\|_{\infty}<\infty .
$$

In the theory of dynamical systems, often Gibbsianness is associated with the SRB measures. These are measures $\mu$ for which there exists a function $\psi:\{-1,1\}^{\mathbb{Z}_{+}} \rightarrow \mathbb{R}$, a constant $\Theta=\Theta(\psi)$, and some finite positive constants $\underline{c}, \bar{c}$ such that

$$
\underline{c} \leq \frac{\mu\left(\omega_{0}^{n}\right)}{\exp \left(\sum_{i=0}^{n} \psi\left(\tau^{i} \omega\right)-(n+1) \Theta\right)} \leq \bar{c}
$$

[ $\tau^{i}$ is the $i$ th iterate of the shift on $\Omega$ ]. In the non-null case, these SRB measures form a strict subset of the one-dimensional Gibbs measures. This can be seen in two ways. First, general Gibbs measures satisfy (2.11) but with the constants $\underline{c}, \bar{c}$ substituted by $o(n)$-functions. Second, the corresponding interactions for the non-null SRB measures must satisfy the condition

$$
\sup _{i \in \mathbb{Z}} \sum_{A \ni i} \operatorname{diam}(A)\left\|\phi_{A}\right\|_{\infty}<\infty
$$

which is stronger than (2.10). 


\section{Main result}

For any $\underline{\omega}=\omega_{-\infty}^{-1} \in \Omega_{-\infty}^{-1}$ and $\bar{\omega}=\omega_{1}^{+\infty} \in \Omega_{1}^{+\infty}$, let

$$
\ell(\underline{\omega})=\min \left\{j \geq 0: \omega_{-j-1}=1\right\} \text { and } m(\bar{\omega})=\min \left\{j \geq 0: \omega_{j+1}=1\right\}
$$

denote the number of 0's before finding the first 1 when looking backward in $\underline{\omega}$ and forward in $\bar{\omega}$, respectively $[\ell(\underline{0})=m(\overline{0})=\infty]$.

Our $g$-function is defined by a converging sequence $\left\{p_{i}\right\}_{i \geq 0}$ of numbers with values in $(0,1)$, satisfying

$$
\inf _{i \geq 0} p_{i}=\epsilon>0 \quad, \quad p_{\infty}=\lim _{i \rightarrow+\infty} p_{i} .
$$

The kernel $P$ is defined on $E \times \Omega_{-\infty}^{-1}$ by

$$
P(1 \mid \underline{\omega})=p_{\ell(\underline{\omega})} \quad \forall \underline{\omega} \in \Omega_{-\infty}^{-1} .
$$

Note that the continuity of the kernel $P$ follows from the fact that

$$
\begin{aligned}
\sup _{\substack{\omega, \sigma \in \Omega_{-\infty}^{-1} \\
\underline{\omega}-1 \\
--k=\sigma_{-k}^{-1}}}|P(1 \mid \underline{\omega})-P(1 \mid \underline{\sigma})| & =\sup _{\substack{\omega_{-\infty}^{-k-1}, \sigma_{-\infty}^{-k-1} \in \Omega_{-\infty}^{-k-1} \\
\omega^{-1}}}\left|P\left(1 \mid 0_{-k}^{-1} \omega_{-\infty}^{-k-1}\right)-P\left(1 \mid 0_{-k}^{-1} \sigma_{-\infty}^{-k-1}\right)\right| \\
& =\sup _{l, m \geq k}\left|p_{l}-p_{m}\right|
\end{aligned}
$$

which goes to 0 since $\left\{p_{k}\right\}_{k \geq 0}$ converges.

This $g$-function is, therefore, regular and, furthermore, in [8] it is proven that there exists a unique stationary chain $\mu$ compatible with $P$ which is the renewal chain with infinitely many 1 's separated by intervals of 0's of random length and having exponential tail distribution. For all practical purposes, this chain is as regular as it can be. Nevertheless, it is not necessary Gibbsian.

Theorem 3.1. There exist choices of the sequence $\left\{p_{i}\right\}_{i \geq 0}$ satisfying (3.2) for which

$$
\mu\left(X_{0}=0 \mid \cdot\right) \text { is essentially discontinuous at } 0_{-\infty}^{+\infty},
$$

where $\mu$ is the (unique and non-null) g-measure compatible with the kernel defined by (3.3).

By Kozlov's theorem [14] this means that the resulting $g$-measure is non-Gibbsian in the statistical mechanical sense, and hence neither in SRB sense.

Proof. The proof consists in the observation that if $\omega$ has $\ell(\underline{\omega})=i$ and $m(\bar{\omega})=j$, then, as we will see in (3.12) below, $\mu\left(X_{0}=0 \mid X_{-m}^{-1}=\omega_{-m}^{-1}, X_{1}^{n}=\omega_{1}^{n}\right)$ is determined by the ratio

$$
\prod_{k=0}^{i-1} \frac{1-p_{k}}{1-p_{k+j}}
$$

Thus, the discontinuity at $0_{-\infty}^{+\infty}$ is equivalent to the existence of a sequence of $p_{k}$ for which this ratio oscillates with $i$ and $j$. The most economical way of achieving this is to define

$$
p_{k}=1-\left(1-p_{\infty}\right) \xi^{v_{k}}
$$


so that

$$
\prod_{k=0}^{i-1} \frac{1-p_{k}}{1-p_{k+j}}=\xi^{\sum_{k=0}^{i-1}\left(v_{k}-v_{k+j}\right)}
$$

The discontinuity is obtained by choosing a sequence $v_{k}$ converging to 0 when $k \rightarrow \infty$, but such that $\sum_{k=0}^{i} v_{k}$ oscillates.

To formalize this idea, let us first provide an explicit expression for the conditional probabilities. By construction, the measure $\mu$ compatible with $P$ has the property that, if $\ell(\underline{\omega})=i \leq m<\infty$ and $m(\bar{\omega})=j \leq n<\infty$

$$
\begin{aligned}
& \mu\left(X_{-m}^{n}=\omega_{-m}^{n}\right) \\
& \quad=\mu\left(X_{-m}^{-i-1}=\omega_{-m}^{-i-1}\right) \mu\left(X_{-i}^{j+1}=\omega_{-i}^{j+1} \mid X_{-i-1}=1\right) \mu\left(X_{j+2}^{n}=\omega_{j+2}^{n} \mid X_{j+1}=1\right)
\end{aligned}
$$

which becomes

$$
\mu\left(X_{-m}^{-i-1}=\omega_{-m}^{-i-1}\right)\left(\prod_{k=0}^{i+j}\left(1-p_{k}\right) p_{i+j+1}\right) \mu\left(X_{j+2}^{n}=\omega_{j+2}^{n} \mid X_{j+1}=1\right)
$$

when $\omega_{0}=0$, and

$$
\mu\left(X_{-m}^{-i-1}=\omega_{-m}^{-i-1}\right)\left(\prod_{k=0}^{i-1}\left(1-p_{k}\right) p_{i}\right)\left(\prod_{k=0}^{j-1}\left(1-p_{k}\right) p_{j}\right) \mu\left(X_{j+2}^{n}=\omega_{j+2}^{n} \mid X_{j+1}=1\right)
$$

when $\omega_{0}=1$. Thus, the finite-volume 2 -sided conditional probability equals

$$
\begin{aligned}
\mu & \left.X_{0}=0 \mid X_{-m}^{-1}=\omega_{-m}^{-1}, X_{1}^{n}=\omega_{1}^{n}\right) \\
& =\frac{\mu\left(X_{-m}^{n}=\omega_{-m}^{-1} 0_{0} \omega_{1}^{n}\right)}{\mu\left(X_{-m}^{n}=\omega_{-m}^{-1} 0_{0} \omega_{1}^{n}\right)+\mu\left(X_{-m}^{n}=\omega_{-m}^{-1} 1_{0} \omega_{1}^{n}\right)} \\
& =\frac{\prod_{k=0}^{i+j}\left(1-p_{k}\right) p_{i+j+1}}{\prod_{k=0}^{i-1}\left(1-p_{k}\right) p_{i} \prod_{k=0}^{j-1}\left(1-p_{k}\right) p_{j}+\prod_{k=0}^{i+j}\left(1-p_{k}\right) p_{i+j+1}} \\
& =\left(1+\frac{p_{i} p_{j}}{\left(1-p_{i+j}\right) p_{i+j+1}} \prod_{k=0}^{i-1} \frac{1-p_{k}}{1-p_{k+j}}\right)^{-1}
\end{aligned}
$$

for all $n \geq i$ and $m \geq j$. Notice that the intermediate equality is symmetric under the interchange $i \leftrightarrow j$; this proves the left-right symmetry of the conditional expectations. Writing the probabilities $p_{k}$ in the form (3.7) this becomes

$$
\begin{aligned}
\mu\left(X_{0}\right. & \left.=0 \mid X_{-i-1}^{-1}=1_{-i-1} 0_{-i}^{-1}, X_{1}^{j+1}=0_{1}^{j} 1_{j+1}\right) \\
& =\left(1+\frac{\left.\left.\left[1-\left(1-p_{\infty}\right) \xi^{v_{i}}\right)\right]\left[1-\left(1-p_{\infty}\right) \xi^{v_{j}}\right)\right]}{\left.\left(1-p_{\infty}\right) \xi^{v_{i+j}}\left[1-\left(1-p_{\infty}\right) \xi^{v_{i+j+1}}\right)\right]} \xi^{\sum_{k=0}^{i-1}\left(v_{k}-v_{k+j}\right)}\right)^{-1} .
\end{aligned}
$$

To conclude we must choose a sequence $v_{k}$ with an oscillating sum, as proposed after (3.8). We choose $\xi \in\left(1,\left(1-p_{\infty}\right)^{-2}\right)$ and consider the sequence $\left\{v_{k}\right\}_{k \geq 0}$ with

$$
v_{k}=\frac{(-1)^{r_{k}}}{r_{k}} \quad \text { with } \quad r_{k}=\inf \left\{i \geq 1: \sum_{j=1}^{i} j \geq k+1\right\} .
$$


The first terms of this sequence are as follows:

$$
-1, \frac{1}{2}, \frac{1}{2},-\frac{1}{3},-\frac{1}{3},-\frac{1}{3}, \frac{1}{4}, \frac{1}{4}, \frac{1}{4}, \frac{1}{4},-\frac{1}{5},-\frac{1}{5},-\frac{1}{5},-\frac{1}{5},-\frac{1}{5}, \ldots
$$

Clearly, $v_{k}$ converges to 0 when $k \rightarrow \infty$, while $\sum_{k=0}^{i} v_{k}$ does not converge as $i$ diverges because it oscillates inside $[-1,0]$. Due to (3.14), there are strictly increasing subsequences

- $\left\{i_{n}^{(1)}\right\}_{n \geq 1}$ such that $\sum_{k=0}^{i_{i}^{(1)}-1} v_{k}=-1$ for any $n \geq 1$;

- $\left\{j_{n}^{(1)}\right\}_{n \geq 1}$, where $j_{n}^{(1)}$ depends on $i_{n}^{(1)}$, such that $\sum_{k=0}^{i_{n}^{(1)}-1} v_{j_{n}^{(1)}+k}=0$ for any $n \geq 1$;

- $\left\{i_{n}^{(2)}\right\}_{n \geq 1}$ such that $\sum_{k=0}^{i_{n}^{(2)}-1} v_{k}=0$ for any $n \geq 1$;

- $\left\{j_{n}^{(2)}\right\}_{n \geq 1}$, where $j_{n}^{(2)}$ depends on $i_{n}^{(2)}$, such that $\sum_{k=0}^{i_{n}^{(2)}-1} v_{j_{n}^{(2)}+k}=0$ for any $n \geq 1$.

Therefore, we have that

$$
\sum_{k=0}^{i_{n}^{(1)}-1}\left(v_{k}-v_{j_{n}^{(1)}+k}\right)=-1
$$

and

$$
\sum_{k=0}^{i_{n}^{(2)}-1}\left(v_{k}-v_{j_{n}^{(2)}+k}\right)=0
$$

Since

$$
\lim _{i, j \rightarrow \infty} \frac{\left.\left.\left[1-\left(1-p_{\infty}\right) \xi^{v_{i}}\right)\right]\left[1-\left(1-p_{\infty}\right) \xi^{v_{j}}\right)\right]}{\left.\left(1-p_{\infty}\right) \xi^{v_{i+j}}\left[1-\left(1-p_{\infty}\right) \xi^{v_{i+j+1}}\right)\right]}=\frac{p_{\infty}}{\left(1-p_{\infty}\right)}
$$

it follows from (3.13)-(3.18) that

$$
\begin{aligned}
\lim _{n \rightarrow \infty} \mu\left(X_{0}=0 \mid X_{-i_{n}^{(\ell)}-1}^{-1}=\right. & \left.1_{-i_{n}^{(\ell)}-1} 0_{-i_{n}^{(\ell)}}^{-1}, X_{1}^{j_{n}^{(\ell)}+1}=0_{1}^{j_{n}^{(\ell)}} 1_{j_{n}^{(\ell)}+1}\right) \\
& = \begin{cases}\frac{\left(1-p_{\infty}\right) \xi}{\left(1-p_{\infty}\right) \xi+p_{\infty}} & \text { if } \ell=1 \\
\left(1-p_{\infty}\right) & \text { if } \ell=2 .\end{cases}
\end{aligned}
$$

Hence $\lim _{i, j \rightarrow \infty} \mu\left(X_{0}=0 \mid X_{-i-1}^{-1}=1_{-i-1} 0_{-i}^{-1}, X_{1}^{j+1}=0_{1}^{j} 1_{j+1}\right)$ does not exist. This contradicts (2.8) and, more specifically, proves the essential discontinuity (3.5).

Acknowledgments. GM is grateful to CNRS for financial support and to EURANDOM for hospitality. SG is supported by a FAPESP fellowship 2009/09809-1. This work is part of USP project Mathematics, computation, language and the brain. We thank Aernout van Enter for his critical reading of the manuscript and his suggestions. 


\section{References}

[1] R. Bowen. Equilibrium states and the ergodic theory of Anosov diffeomorphisms, Lecture Notes in Mathematics, Vol. 470, Springer-Verlag, Berlin, 1975. MR442989

[2] R. L. Dobrushin, The description of a random field by means of conditional probabilities and conditions of its regularity. Theory Probab. Appl., 13 (1968) 197-224.

[3] A.C.D. van Enter, R. Fernández, and A.D. Sokal, Regularity properties and pathologies of position-space renormalization-group transformations: scope and limitations of Gibbsian theory, J. Stat. Phys. 72 (1993) 879-1167. MR1241537

[4] A.C.D. van Enter and E. Verbitskiy, Erasure entropies and Gibbs measures, Markov Process. Related Fields 16 (2010) 3-14. MR2664333

[5] R. Fernández, Gibbsianness and non-Gibbsianness in Lattice random fields, In: Mathematical statistical physics, (A. Bovier, A.C.D. van Enter, F. den Hollander and F. Dunlop eds.), 731799, Elsevier B. V., Amsterdam, 2006. MR2581896

[6] R. Fernández and G. Maillard, Chains with complete connections and one-dimensional Gibbs measures, Electron. J. Probab. 9 (2004) 145-176. MR2041831

[7] R. Fernández and G. Maillard, Construction of a specification from its singleton part, ALEA 2 (2006) 297-315. MR2285734

[8] S. Gallo, Chains with unbounded variable length memory: perfect simulation and visible regeneration scheme, Adv. in Appl. Probab. 43 (2011) 735-759.

[9] H.-O. Georgii, Gibbs Measures and Phase Transitions, de Gruyter Studies in Mathematics, Vol. 9, Walter de Gruyter \& Co., Berlin, 1988. MR956646

[10] T. E. Harris, On chains of infinite order, Pacific J. Math., 5 (1955) 707-24. MR75482

[11] S. Kalikow, Random Markov processes and uniform martingales, Isr. J. Math., 71 (1990) 33-54. MR1074503

[12] M. Keane, Strongly mixing g-measures, Inventiones Math. 16 (1972) 309-324. MR310193

[13] G. Keller, Equilibrium states in ergodic theory, London Mathematical Society Student Texts, Vol. 42, Cambridge University Press, Cambridge, 1998. MR1618769

[14] O. K. Kozlov, Gibbs description of a system of random variables, Probl. Inform. Transmission, 10 (1974) 258-265. MR467970

[15] O. E. Lanford III and D. Ruelle, Observables at infinity and states with short range correlations in statistical mechanics, Comm. Math. Phys., 13 (1969) 194-215. MR256687

[16] O. Onicescu and G. Mihoc, Sur les chaînes statistiques, C. R. Acad. Sci. Paris, 200 (1935) 511-512.

[17] D. Ruelle, Thermodynamic formalism. Encyclopedia of Mathematics 5, Addison Wesley, NewYork, 1978. MR511655 